\title{
A correlation network approach to metabolic data analysis for tomato fruits
}

\author{
Remco Ursem · Yury Tikunov · Arnaud Bovy • \\ Ralph van Berloo $\cdot$ Fred van Eeuwijk
}

Received: 16 May 2007/ Accepted: 12 February 2008/Published online: 11 March 2008

(C) The Author(s) 2008

\begin{abstract}
Network analysis of correlations between abundances of metabolites across tomato genotypes can help in unraveling the biological basis of organoleptic variation in tomato. We illustrate how to construct and interpret simple correlations networks using metabolic data collected on a diverse set of tomato genotypes. Various types of correlations are calculated and displayed in the form of networks. Interpretations on the basis of network analyses are compared to interpretations following principal components analysis.
\end{abstract}

Keywords Correlation network - Metabolomics · Network analysis · Principal component analysis · Tomato

R. Ursem · Y. Tikunov · A. Bovy · R. van Berloo . F. van Eeuwijk

Centre for BioSystems Genomics, P.O. Box 98, 6700 AB Wageningen, The Netherlands

R. Ursem $(\bowtie) \cdot$ R. van Berloo

Laboratory of Plant Breeding, Wageningen University, P.O. Box 386, 6700 AJ Wageningen, The Netherlands e-mail: remco.ursem@wur.nl

\section{Y. Tikunov $\cdot$ A. Bovy}

Plant Research International, 6700 AA Wageningen, The Netherlands

F. van Eeuwijk

Biometris, P.O. Box 100, 6700 AC Wageningen,

The Netherlands

\section{Introduction}

The Center for BioSystems Genomics (CBSG) is a joint venture in the field of plant genomics of breeding companies, biotech companies, research institutes and universities in the Netherlands. At the web site (http:// www.cbsg.nl) it is stated that 'The consortium aims at developing knowledge for the improvement of food and non-food crops with reduced environmental impact and enhanced consumer quality.' An important part of CBSG concentrates on tomato quality with the objective of developing a better understanding of the biological factors involved in tomato taste, with special attention for the underlying genetics. The ultimate goal of the CBSG tomato quality program is the development of a marker assisted improvement strategy for quality traits. The empirical basis of the current research on tomato quality is formed by various types of observations on a diverse set of tomato consisting of 94 genotypes, mostly cultivars and hybrids. Within the phenotypic data, a prominent place is given to a set of metabolites. Before trying to identify the genetic basis of the variation in metabolite levels by marker-trait association analyses, we decided to investigate the patterns of variation and covariation (correlation) in the metabolites by network visualization. Network construction and representation techniques provide an interesting complement to more classical multivariate methods as described in their application to metabolites in Graffelman and van Eeuwijk (2005) and van Berloo et al. (submitted). We expect that metabolite 
patterns as identified by network techniques can eventually be better related to molecular marker variation than the variation in individual metabolites.

Metabolite correlations are believed to provide a 'fingerprint' of the underlying biophysical system (Steuer et al. 2003b; Steuer et al. 2003a; Weckwerth et al. 2004; Morgenthal et al. 2006). Elucidating the origin of metabolite correlations will give us insight into biochemical processes and their regulation (Camacho 2005; Steuer 2006).

To illustrate the application of network methodology to metabolite data collected within the CBSG tomato quality program, a selection out of a larger set of scored metabolites was made, consisting of several volatile organic compounds (secondary metabolites that evaporate at normal temperatures), and some primary metabolites-sugars and organic acids. All substances were known or expected to play a role in tomato taste sensation (Baldwin et al. 2000; Tandon et al. 2003; Baldwin et al. 2004; Krumbein et al. 2004; Ruiz et al. 2005).

\section{Material and methods}

\section{Tomato cultivars}

The collection of tomato cultivars used within the CBSG tomato quality project was selected to be a diverse set with respect to tomato quality traits, plant characteristics and genotypic background. All cultivars were greenhouse cultivars provided by the five international breeding companies involved in the project, and almost all were F1 hybrids. In this study we used data of 94 cultivars for which there was a complete dataset consisting of AFLP marker scores, metabolic profiles, organoleptic trait evaluations, plant and fruit morphology measurements and consumer appreciation assessments. The cultivars were classified by eye into three different types of tomato, labelled as round, beef and cherry. The three groups made up roughly 50,25 , and $25 \%$ of the total collection, respectively. A priori, there was no reason to believe that metabolite patterns should differ between tomato types. The cultivars were evaluated in three greenhouse experiments over a 2-yearperiod. Each experiment consisted of a randomized complete block design with three replicates. Individual plots consisted of three plants.
Metabolic profiling

For a given experiment, metabolic profiling of cultivars was based on pooled fruit samples, where the pooling arose from combining fruits from the three replicates of the experiment. Samples for round and beef tomatoes contained 12 red ripe fruits, while samples for cherry tomatoes contained 18 fruits. Individual tomatoes from individual replicates could not be identified anymore once the pooled samples were constructed. Metabolic assessments thus pertained to the pooled samples, one pooled sample for each cultivar in an experiment. Volatile compounds of red ripe fruit material were relatively quantified and identified using Gas Chromatography/Mass Spectrometry (GC/MS) (for details, see Tikunov et al. 2005). Sugars and organic acids were profiled using GC/MS as described in Roessner-Tunali et al. (2003). The volatiles were measured once in the first experiment, twice in the second experiment, and once in the third experiment. The two metabolic assessments in the second experiment concerned a duplicate metabolic sample preparation (technical replicate) and not a biological duplication, i.e., the same pooled fruit samples were used for both metabolic assessments. The sugars and organic acids were measured once in all three experiments.

Estimation of genotypic means, heritability and genetic correlation

All metabolites were $\log _{10}$ transformed before further analysis to improve normality. Linear mixed models were used to estimate genotypic means. We used these models to estimate mean trait values per genotype, taking into account other factors that might influence individual measurements like environment and measuring day. Genotypic metabolite means were estimated with the following mixed model for metabolite abundance:

$y_{i j k}=\mu+d_{k}+g_{i}+(g e)_{i j}+\varepsilon_{i j k}$

where $d_{k}(k=1 \ldots 41)$ stands for measurement day; a compound effect of experiment, replication and measurement day, with measurement day nested within replication, and replication nested within experiment. The effect $g_{i}(i=1 . . .94)$ represents the main genotype effect, while $(g e)_{i j}$ is the effect for the interaction of genotype $i$ and experiment $j(j=1 \ldots 3)$. The terms $d_{k}$ and $g_{i}$ were fixed terms, and $(g e)_{i j}$ and the error term, $\varepsilon_{i j k}$, 
were random. We realize that our linear mixed model may look somewhat unconventional in the sense that we include a random genotype by experiment interaction on top of two fixed main effects. First, the reason to include a fixed day effect was that we found that for almost all traits there was reason to correct the genotypic means for the effects of experiment, replicate and measurement day. However, the relative sizes of those effects differed very strongly between traits. Fitting the three experiments or the two replicates within the second experiment as random terms did not make sense due to the low number of levels. The only term representing 'environmental' variation that would classify for possibly being random was day within replicate within experiment. However, as we found that these day effects looked sometimes very far from normal, we preferred to take the day effects fixed. Furthermore, we felt uneasy to recover genotype information from the day totals, which was another reason to take these effects fixed. As we were interested in the genotypic means as such we decided to choose the main effects of genotypes fixed. For the generation of genotypic means, we saw the genotype by experiment effects as nuisance parameters and thus we took this interaction random. Random genotype by replicate within experiment effects were absent or so small as to be ignorable.

To estimate broad-sense heritability, $h^{2}$, or better, repeatability, of each metabolite, we adapted the above given linear mixed model by changing the status of the genotypic main effects from fixed to random. The heritability was then calculated by introducing the corresponding estimated variance components in the following equation, which will provide a rough estimator for the heritability.

$h^{2}=\frac{\sigma_{g}^{2}}{\sigma_{g}^{2}+\frac{\sigma_{g e}^{2}}{n_{e}}+\frac{\sigma_{\varepsilon}^{2}}{n_{e} n_{r}}}$.

Here $\sigma_{g}^{2}, \sigma_{g e}^{2}$ and $\sigma_{\varepsilon}^{2}$ are the variance between genotypes, the variance for genotype by experiment interaction, and the residual variance. Further, $n_{e}=3$, is the number of experiments, and $n_{r}$ is the number of replications within experiments, for which we took 1.2 for the volatiles, the harmonic mean of the number of replications across the three experiments, 1, 2, and 1, respectively. For sugars and acids only one replication was taken per experiment, so then $n_{r}=1$.

Genetic correlations between metabolites can be estimated in various ways. An elegant, but elaborate method is to fit multi-trait mixed models like in Malosetti et al. (2008). For exploration purposes, a quick, although less accurate method for calculating genetic correlations can be used:

$r_{g}=\frac{r_{p}}{h_{x} h_{y}}$

(Burdon 1977; Falconer et al. 1996), where $r_{g}$ is the genetic correlation, $r_{p}$ the phenotypic correlation, and $h_{x}$ and $h_{y}$ the square root heritabilities of the concerned metabolites. This method follows directly from the text book formulation of the phenotypic correlation between two traits $x$ and $y$ as $r_{p}=h_{x} h_{y} r_{g}+e_{x} e_{y} r_{e}$ (Falconer et al. 1996), with $e_{x}=\sqrt{1-h_{x}^{2}} ; e_{y}=\sqrt{1-h_{y}^{2}}$ and $r_{e}$ as the correlation between the traits due to common micro-environmental influences. When we assume that $r_{e}=0$ because of our experimental and randomization procedures, we arrive at the above expression for estimation of genetic correlations. This estimation approach can produce estimates exceeding one. Genetic correlations exceeding one were set at one.

To study metabolite relations while correcting for differences due to tomato type, we also estimated tomato-type corrected correlations between metabolites. These type-corrected correlations were estimated by regression of the genotypic means on tomato type (cherry/non-cherry) followed by calculation of correlations between the residual metabolite abundances.

Besides the above mentioned correlations, we also calculated partial correlations, correlations between metabolites conditional on all other metabolites, i.e. correlations between pairs of metabolites that remain after removal of covariation due to joint dependence on third metabolites. Partial correlations were calculated from the inverse of the correlation matrix as follows. Let $a_{i j}$ be the $i j$-th element of the inverse of the correlation matrix, then the partial correlation, $r_{i j}^{\text {partial }}$, between the metabolites $i$ and $j$ is calculated as $r_{i j}^{\text {partial }}=\frac{-a_{i j}}{\sqrt{a_{i i} a_{j j}}}$. With these partial correlations we try to determine to what extend metabolite correlations are direct (not depending on other metabolites in the network).

Distributions of traits

As a first data exploration and to compare relative abundance of the different metabolites in our collection of tomatoes, boxplots were constructed for all metabolites. With these boxplots we can compare 
quartiles and ranges, and get an idea about trait distributions in the tomato collection as a whole.

Principal component analysis

We used principal component analysis (PCA) to study patterns of variation in sets of metabolites. The central idea of PCA is to reduce the dimensionality of a data set consisting of a large number of interrelated variables, while retaining as much as possible of the variation. This is achieved by transforming to a new set of variables, the principal components (PCs), which are uncorrelated, and which are ordered so that the first few retain most of the variation present in the full set of the original variables (Jolliffe 2002). All statistical analyses were done on $\log _{10}$ transformed data and as explained above, genotypic means were produced from mixed models applied to the transformed data. Use of the log transform already scales variation in different traits to comparable levels and thus we applied PCA to the genotypic means on the covariance scale, so that we still could discriminate between metabolites with large and small magnitudes of variation, information that would be lost when applying PCA to standardized data. The loadings (weights) of the metabolites on the principal components indicate which traits show similar, opposite or distinct variation. The PCA output was used to construct biplots to visualize trait variation in relation to the principal components and to show how the tomatoes in our collection were distributed over this part of data space (Graffelman and van Eeuwijk 2005). Biplots were scaled in such way that sum of squares of the metabolite loadings was equal to the corresponding eigenvalue. Biplots help in the identification of clusters of metabolites that may be related by function or regulation. Alternatively, when clustering of tomato genotypes is observed, the metabolites that cause this clustering can be identified.

\section{Correlation network analysis}

Correlation networks offer another way to investigate patterns in the pairwise correlations between metabolites. Networks consist of a set of nodes connected by a system of edges. In correlation networks, the nodes are determined by the metabolites, while the edges represent the correlations between the metabolites. The stronger the correlation between a pair of metabolites, the thicker the line connecting those metabolites in the network graph.
With correlation network plots, not only clusters of metabolites can be identified, but this visualization technique also makes it possible to see how clusters of metabolites are connected. The network plots can give us insight into the functional and regulational relations between metabolites, by comparing metabolic correlation networks to known biochemical pathways.

For the construction of the metabolic networks, we used Pearson product-moment correlations between genotypic means of pairs of metabolites. As a first variation on this basic approach, we also calculated and plotted partial correlations between metabolites after correction for tomato type. Furthermore, we estimated and plotted genetic correlations between metabolites. By means of partial correlations we investigated the unique parts of relations between metabolites, i.e., the parts of correlations that are independent of other metabolites in the network.

All networks were constructed using the Pajek graph drawing software (Batagelj and Mrvar 2003, URL: http://vlado.fmf.uni-lj.si/pub/networks/pajek/), but we emphasize that we only used this package to fine tune the visual layout of the vertices (metabolites) and edges (correlations) that represent the correlation matrix in the plane. To compare the various types of correlation networks we kept the positioning of the vertices (metabolites) the same in all graphs, so only the connecting lines (correlations) change. For all pairwise correlations, $P$-values were calculated to determine significance. The $P$-values were converted into $q$-values, a measure of significance in terms of the false discovery rate (Benjamini et al. 1995), using a procedure in the R-package (Storey et al. 2003). In this way significance of correlations was determined, while correcting for multiple comparisons (300 pairwise correlations). The $q$-value approach is less conservative and more powerful than the Bonferroni correction method (Storey et al. 2003). Correlations were called significant when $q \leq 0.05$. Only the significant correlations were used in the construction of networks. To improve interpretation of the correlation networks, the two highest correlations for each metabolite (when appropriate) were drawn in black, while all others were drawn in grey.

\section{Discriminant analysis}

The unsupervised analyses described above were extended with a supervised one. We used discriminant 
analysis (Dillon et al. 1984) to investigate metabolic variation within and between the different types of tomato (beef, round and cherry). Data on all 25 metabolites in all 94 genotypes was used. Discriminant analysis finds linear combinations of metabolites that maximize the ratio of the between group variance to the averaged within group variance, thereby producing functions of the metabolites that can be used to discriminate between the tomato groups. Estimating the correlation between metabolic abundances and discriminant function scores provides an indication of which metabolites discriminate between tomato types.

\section{Results}

\section{Distributions of traits}

Boxplots were constructed with the genotypic means for metabolite abundance (Fig. 1). Box width in Fig. 1 is proportional to estimated heritability of the metabolite and gives an idea about the genetic basis and breeding potential. The boxplots show strong variation between metabolites with respect to median abundance and range. Metabolites with a wide range and high heritability (repeatability) are the most amenable to improvement by breeding, where potential tomato quality improvement depends on the relation between metabolites and the organoleptic traits. In Fig. 1 we can see that 2-methoxyphenol and methylsalicylate comply with the requirements of large variation and high heritability. High heritability with somewhat less variation was found for phenylethanol. Next, there was a group of metabolites with reasonably high heritability, but with intermediate variation: 2-methylbutanal, 3-methylbutanol, 2-methylbutanol, 2-isobutylthiazol and phenylacetaldehyde.

Relative abundances of volatiles were difficult to compare to those of sugars and acids because of differences in protocol, where the protocol for the latter groups included a derivatisation step (see Tikunov et al. 2005 for details).
Fig. 1 Boxplots of metabolite data $\left(\log _{10}\right.$ transformed), showing metabolite variation within a collection of 94 tomato cultivars. Box widths (vertical) represent estimated percentage heritability of metabolites, ranging from 12.5 (glutamic acid) to $95.1 \%$ (methylsalisylate). Horizontal lines separate volatiles, sugars and acids

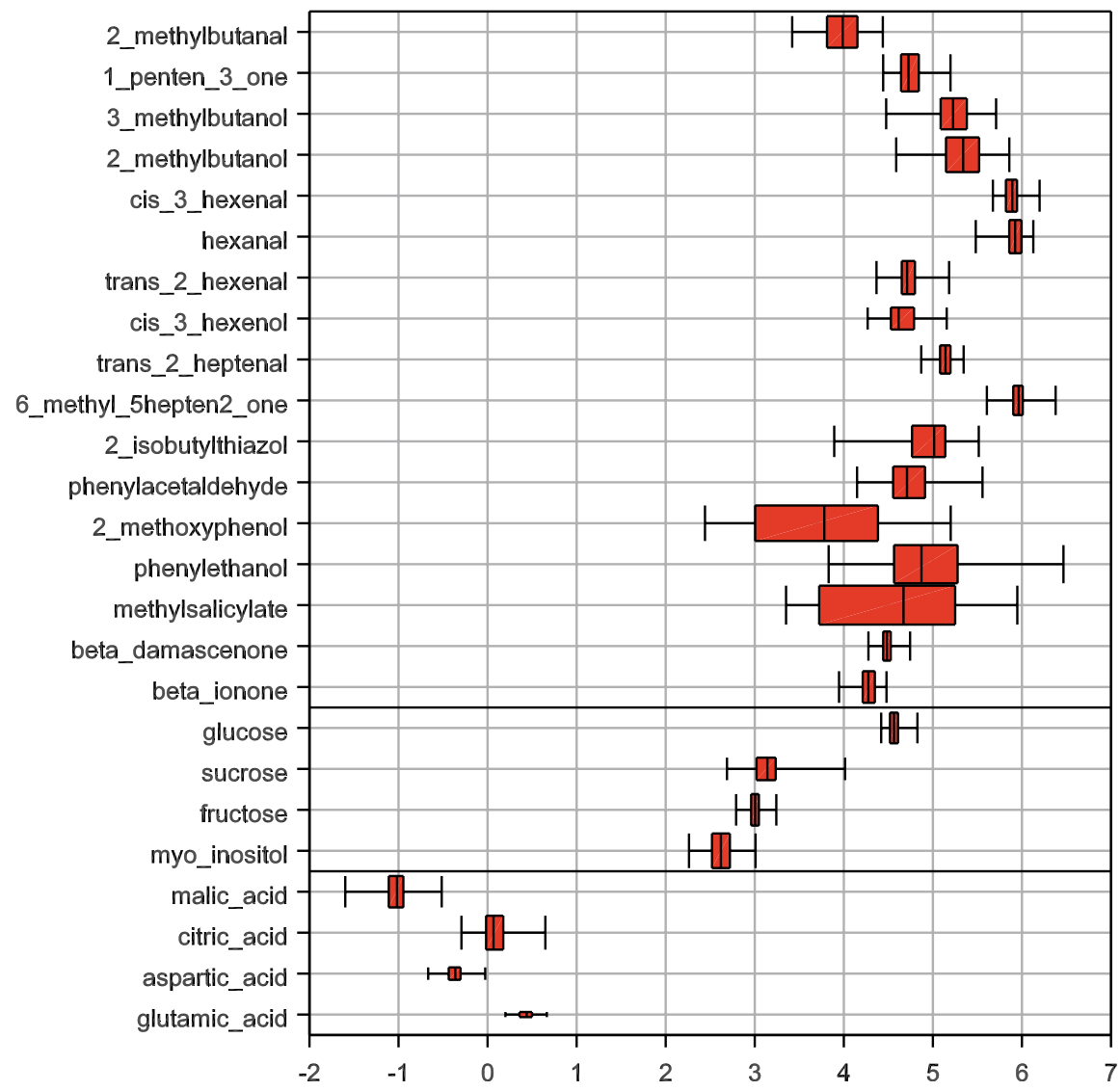


Principal component analysis

From the PCA on metabolite data biplots were constructed. In these biplots, the first three principal axes are shown, axis two against one in Fig. 2 and axis three against two in Fig. 3. The first axis shows large variation in methoxyphenol and methylsalicylate within the tomato collection. These metabolites are known to be phenylpropanoid volatiles (for an overview of tomato volatile precursors and pathways, see Buttery et al. 1993; Baldwin et al. 2000; Tikunov et al. 2005). Other metabolites do not seem to contribute to the first principal axis. On the second principal axis the cherry type tomatoes are clearly separated from the round and beef type tomatoes. On this second axis we see large variation in phenylethanol, some variation in phenylacetaldehyde, sucrose and malic acid. Phenylacetaldehyde, sucrose and especially phenylethanol have higher concentrations in cherry tomatoes, while malic acid is less abundant. Phenylethanol and phenylacetaldehyde are phenolic volatiles. The third principal axis is mainly based on four other metabolites, namely 3-methylbutanol, 2-methylbutanol, 2-methylbutanal, and 2-isobuthylthiazol. These metabolites are (iso)leucine derived volatiles.
On the first and third axis no separation of tomato types is visible. The first three principal axes explain, respectively, 53.8, 20.2 and $8.5 \%$ of the total metabolite variation measured.

\section{Correlation network analysis}

\section{A network of correlations between genotypic means}

Figure 4 shows the network that was constructed from all significant pairwise correlations between metabolites. As expected, this correlation network shows similar trends as the biplots in Figs. 2 and 3, with links between metabolites in the network of Fig. 4 coinciding with groups of metabolites loading on the same principal component, or axis, in the Figs. 2 and 3. For example, methoxyphenol and methylsalicylate were strongly connected and the same holds for the phenolic metabolites (phenylethanol and phenylacetaldehyde) and the (iso)leucine derived metabolites (2- and 3-methylbutanol, 2methylbutanal). Besides these expected connections, other clusters of highly connected metabolites can be identified. The network shows a hexanal, trans-2heptanal, $\beta$-ionone cluster, a cluster consisting of
Fig. 2 Biplot showing the first axis (horizontal, $53.8 \%$ of total variation) and second axis (vertical, $20.2 \%$ of total variation) of a principal component analysis on $\log _{10}$ transformed metabolite abundance in 94 tomato cultivars. Diamonds represent metabolites, circles represent tomato cultivars. Tomato types are indicated by yellow (cherry), blue (beef) and red (round) phenylethanol

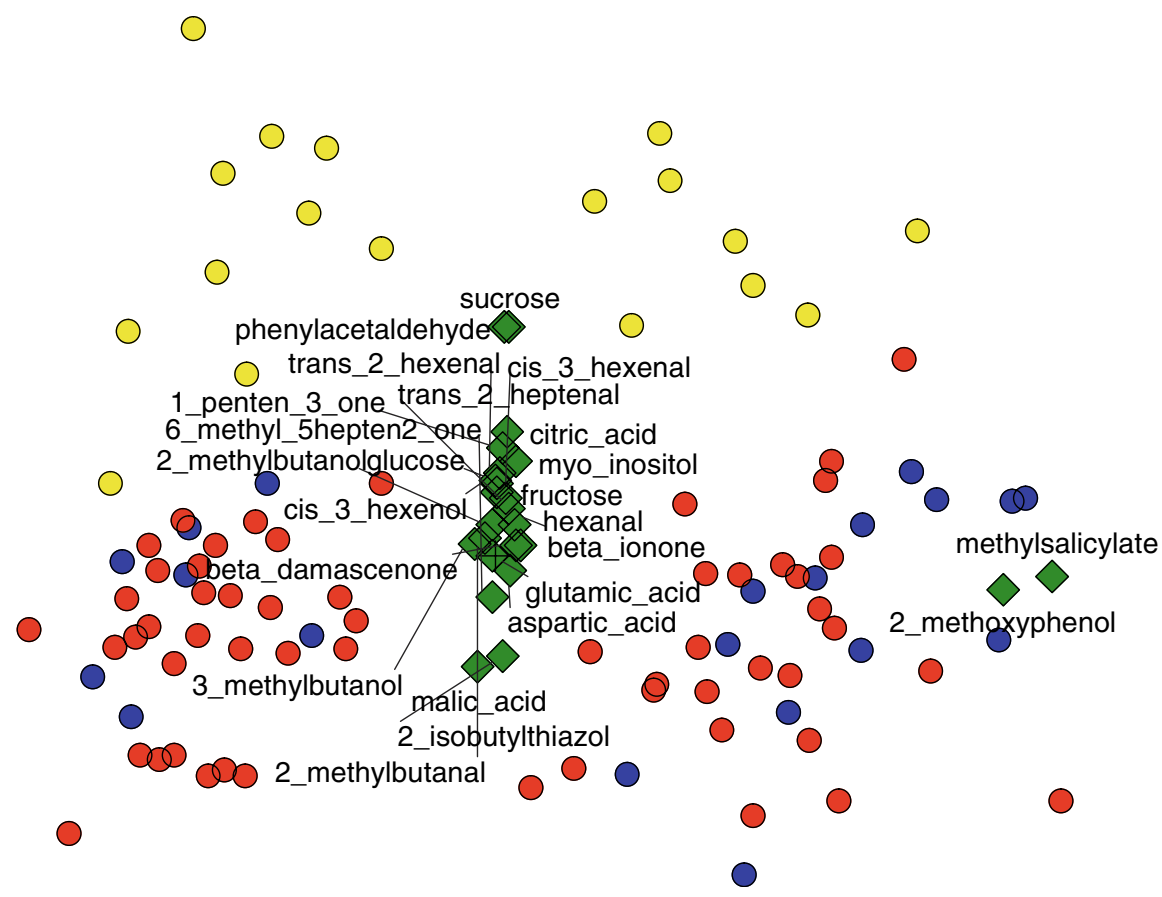


Fig. 3 Biplot showing the second axis (horizontal, $20.2 \%$ of total variation) and third axis (vertical, $8.5 \%$ of total variation) of a principal component analysis on $\log _{10}$ transformed metabolite abundance in 94 tomato cultivars. Diamonds represent metabolites, circles represent tomato cultivars. Tomato types are indicated by yellow (cherry), blue (beef) and red (round)

Fig. 4 Correlation network based on $\log _{10}$ transformed metabolite abundance in 94 tomato cultivars. Nodes represent metabolites, lines their pairwise correlations. Only significant $(q<0.05)$ correlations are drawn. Line thickness indicates correlation strength. Dotted lines represent negative correlations. The two strongest connections per metabolite are depicted in black, all others in grey 3_methylbutanol

2_methylbutanol

2_methylbutanal

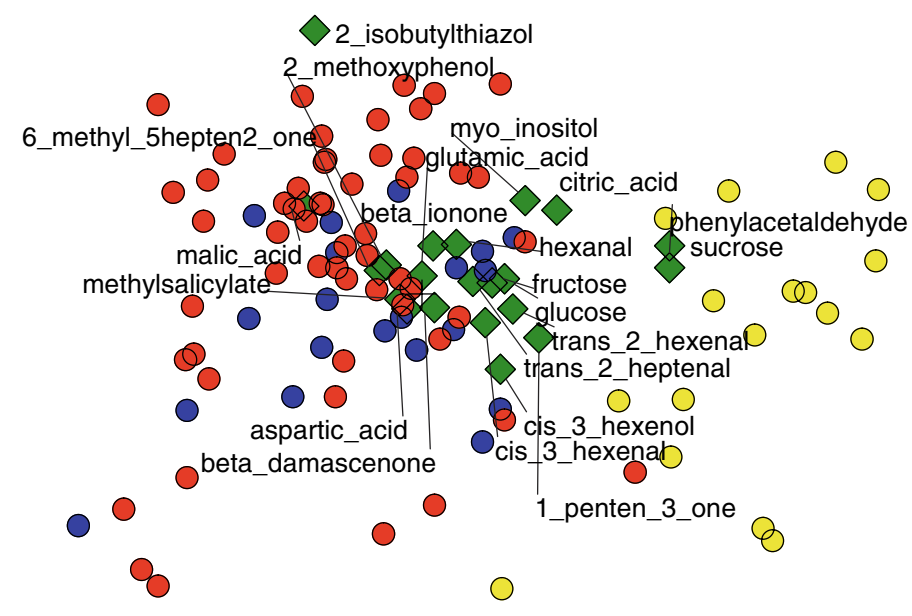

phenylethanol

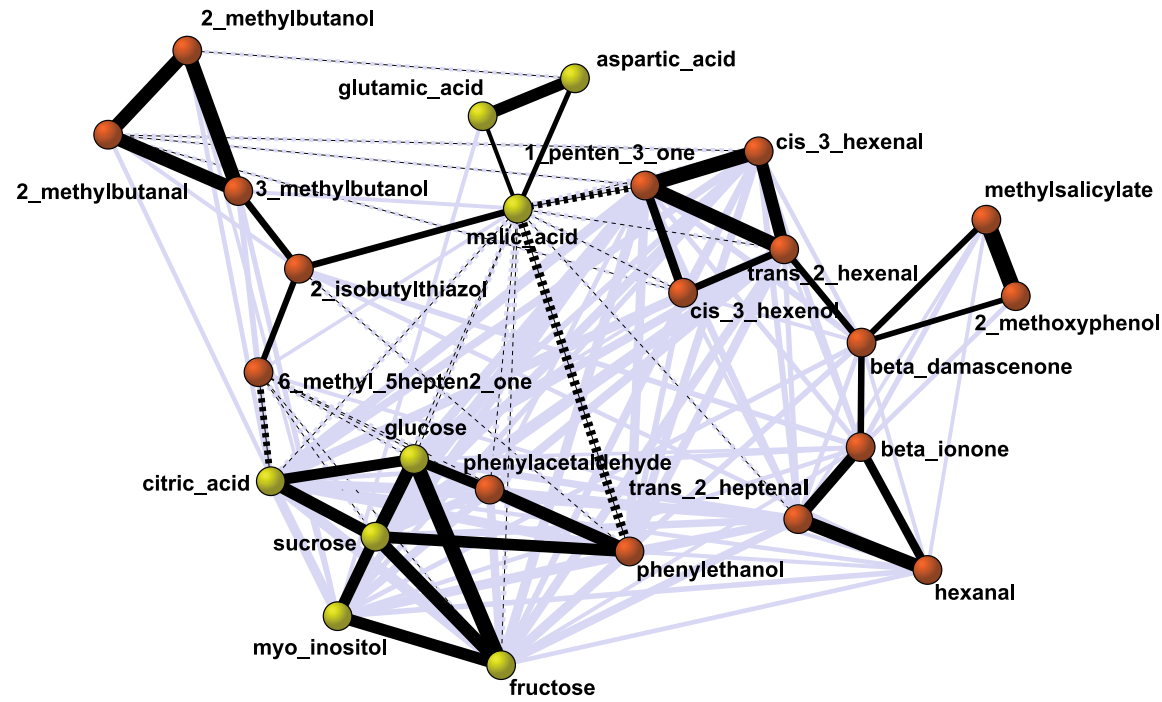

trans-2-hexenal, cis-3-hexenal, 1-penten-3-one, cis3-hexenol (all lipid derived volatiles), a glutamic- and aspartic-acid (amino acids) connection, and a cluster containing all sugars, citric acid and the earlier mentioned phenylethanol, phenylacetaldehyde connection. In the background, many, sometimes strong, connections between the sugars, citric acid, and the 1penten-3-one, trans/cis hexenal cluster are visible. Figure 4 clearly shows that many metabolites were significantly correlated to many others and, furthermore, indicates that both the highlighted, strongest relations and the background relations should be taken into account when drawing conclusions.

\section{Networks of partial and genetic correlations}

The interpretation of relations between metabolites through the use of networks can be made more 
meaningful by working not only with standard correlations between metabolites, but also with partial correlations between metabolites after correction for particular genetic or environmental disturbing factors. Further variations are possible when considering genetic correlations instead of phenotypic correlations, and when looking at correlations within particular groups of genotypes. Figures 5-8 show networks based on partial correlations adjusted for tomato type (Fig. 5), genetic correlations (Fig. 6), correlations within the cherry subpopulation (Fig. 7), correlations within the non-cherry subpopulation (Fig. 8) and partial correlations (Fig. 9), respectively. The positions of the vertices representing the metabolites in Figs. 5-8 were fixed to their positions from
Fig. 4 to focus on changing edges in the graphs (the metabolite relations).

Comparing the network in Fig. 4 to the other networks shows the main connections described earlier to be consistent over all types of networks, but simultaneously reveals many shifts in the levels of the correlations. Some examples of major shifts are the mainly non-cherry driven links of citric acid and 2isobutylthiazol to the (iso)leucine cluster, the cherry driven link between the sugars and the pentenone, cis/ trans hexenal cluster. Furthermore, the connection of phenylacetaldehyde and phenylethanol to the sugars and trans-2-heptanal was much stronger within the cherry tomatoes than the non-cherry tomatoes. In the cherry subpopulation, the phenylpropanoids (methylsalicylate,
Fig. 5 Tomato-type corrected correlation network based on $\log _{10}$ transformed metabolite abundance in 94 tomato cultivars corrected for the cherry versus non-cherry type tomato contrast. Representation is identical to representation in Fig. 4.
Fig. 6 Genetic correlation network based on $\log _{10}$ transformed metabolite abundance in 94 tomato cultivars. Representation is identical to representation in Fig. 4
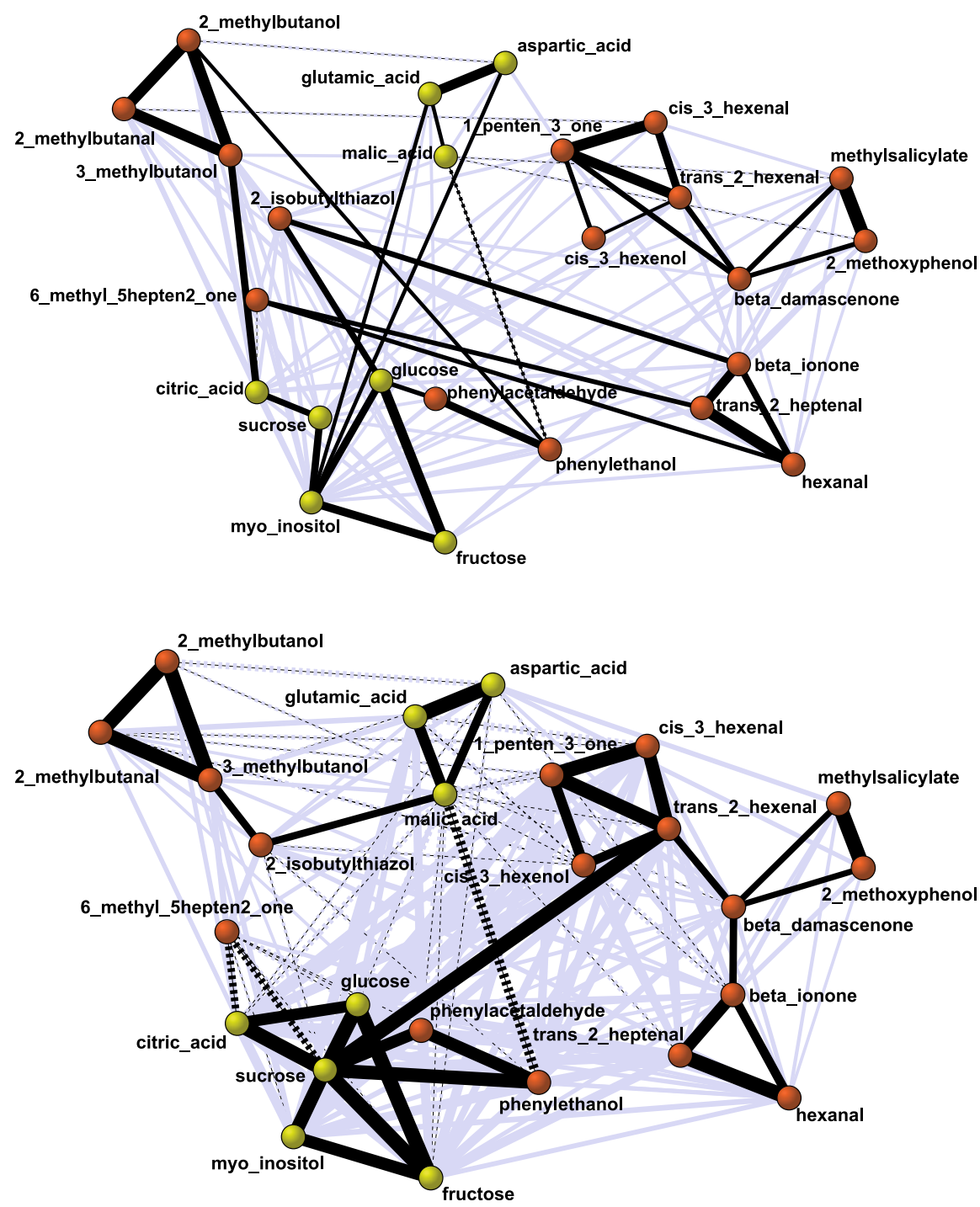
Fig. 7 Correlation network based on $\log _{10}$ transformed metabolite abundance in cherry tomato cultivars. Representation is identical to representation in Fig. 4

Fig. 8 Correlation network based on $\log _{10}$ transformed metabolite abundance in beef and round tomato cultivars. Representation is identical to representation in Fig. 4
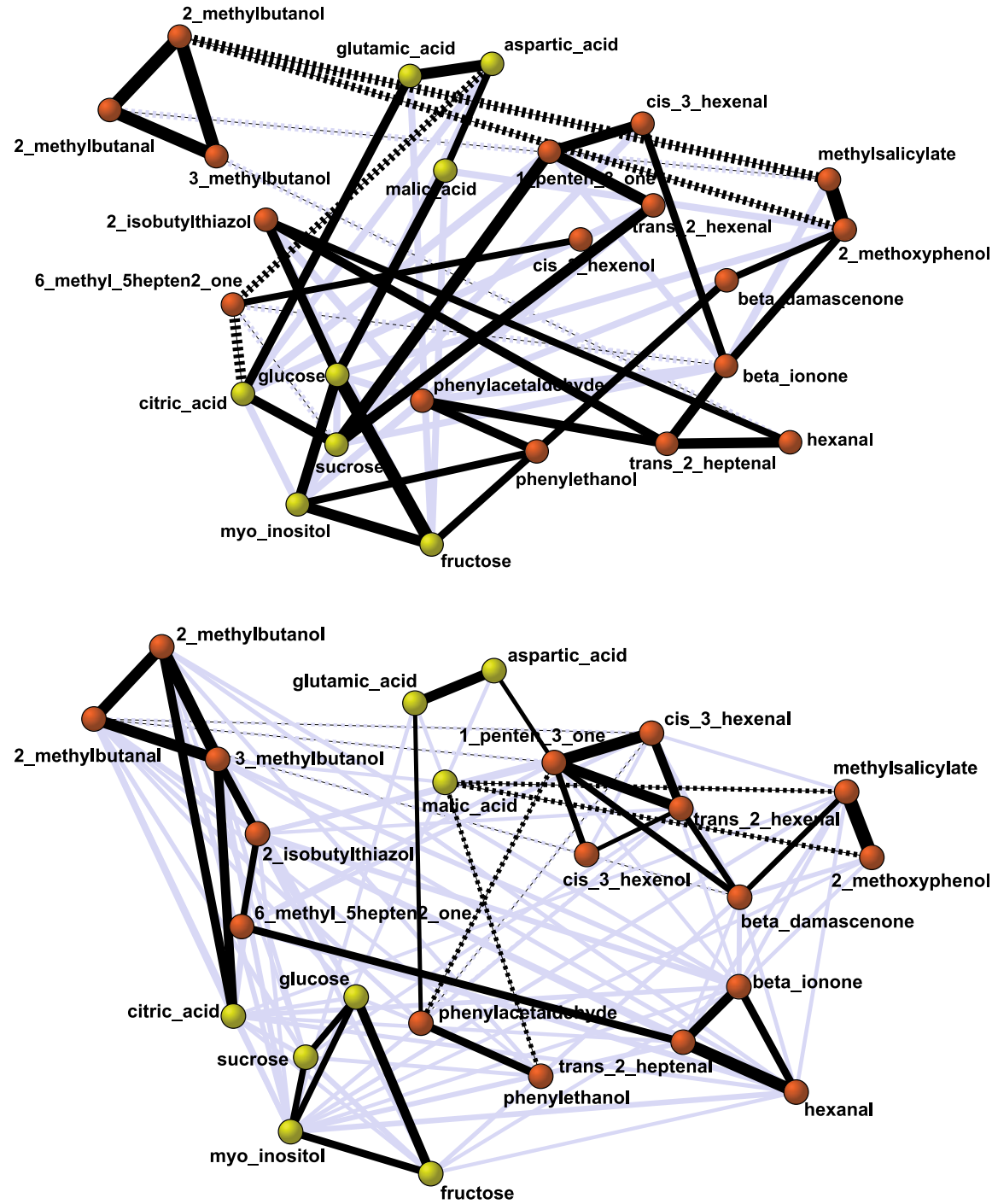

Fig. 9 Partial correlation network based on $\log _{10}$ transformed metabolite abundance in 94 tomato cultivars. Representation is identical to representation in Fig. 4

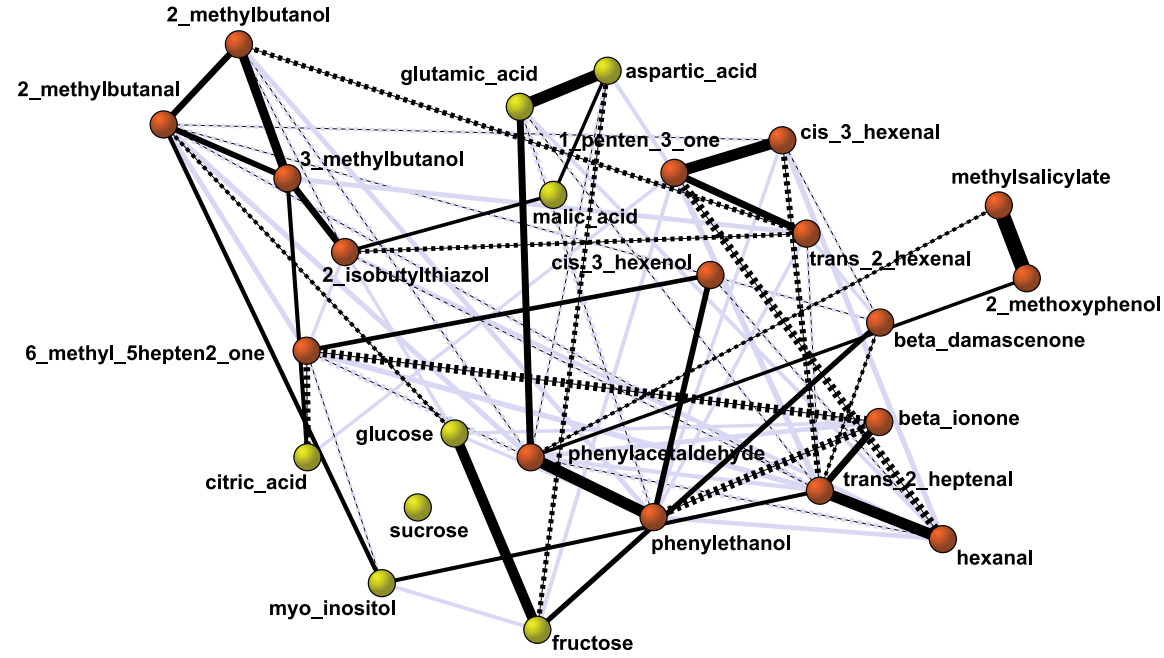


2-methoxyphenol) had a high negative correlation with 2-methylbutanol, while in the non-cherry subpopulation the phenylpropanoids were negatively correlated to malic acid.

Figure 5 shows the tomato-type corrected correlation network, correlations between the residuals of the metabolite genotypic means after regression on the cherry-non-cherry contrast. Major differences between networks for corrected (Fig. 5) and uncorrected (Fig. 4) metabolites are the weaker connected sugars/ phenolic volatiles cluster and the lower correlations of this cluster with the hexanal/pentenone cluster and trans-2-heptenal. Furthermore, malic acid loses part of its negative correlations with the sugars/phenolic volatiles cluster and 2-isobutylthiazol gains a strong positive correlation to glucose in the tomato type corrected network of Fig. 5. This makes sense since most sugars, citric acid and the phenolic volatiles had a higher relative abundance and malic acid and 2-isobutylthiazol had lower relative abundance in cherry compared to beef and round tomatoes.

In the network based on estimated genetic correlations (Fig. 6), the central role for sucrose is even stronger than in the networks based on phenotypic correlations (Fig. 4) and partial phenotypic correlations (Fig. 5). Sucrose is the highest correlated metabolite for citric acid, all measured sugars, the phenolic volatiles and trans-2-hexanal in the genetic network of Fig. 6 (Some qualification is necessary here in that sucrose and the amino acids had a relatively low estimated heritability, which may lead to an over-emphasis on the genetic correlations involving these metabolites due to the method we used to estimate genetic correlations).

The network based on partial correlations in Fig. 9 shows that many of the main metabolite relations, the strong correlations that turn up in all networks, seem to be direct relations, i.e., relations that are largely independent of the variation in the other metabolites. Major exception to this finding is the sugar cluster, where all correlations but the glucose-fructose and a small fructose-myo-inositol correlation are lost. Furthermore some other metabolite relations turn out to be indirect, depending on a third metabolite that connects the two. One example is the trans-2-hexenal relation with cis-3-hexenal via 1-penten-3-one, and the hexanal relation with $\beta$-ionone via trans-2-heptenal. In addition new relations can be found, like the positive direct relation between hexanal and cis-3-hexenal that goes together with a negative direct relation between hexanal and 1-penten-3-one, a metabolite that shows a strong direct relation with cis-3-hexenal.

Discriminant analysis

A clear separation between cherry and non-cherry tomatoes was found by discriminant analysis (Fig. 10). Metabolites discriminating between cherry and noncherry tomatoes to a large extent also contributed to the second principal axis in the PCA analysis (compare Figs. 2 and 3 with Table 1a). Table $1 \mathrm{a}$ and $\mathrm{b}$ show the correlations between metabolite abundances and discriminant function scores, 1 a for discriminant function 1 and $1 \mathrm{~b}$ for discriminant function 2 . The tables are sorted on absolute correlation. The separation between round and beef tomatoes is much less clear (Fig. 10). 2isobutylthiazol, 6-methyl-5hepten2_one and methylsalicylate are the most important metabolites to discriminate between round and beef typed tomatoes and have significantly different means ( $t$-test, $P<0.05)$ in round and beef tomatoes. Figure 11 shows a boxplot of 2-isobutylthiazol for all three tomato types. Both discriminant functions were significant, function 1 and 2 described 91 and $9 \%$ of the

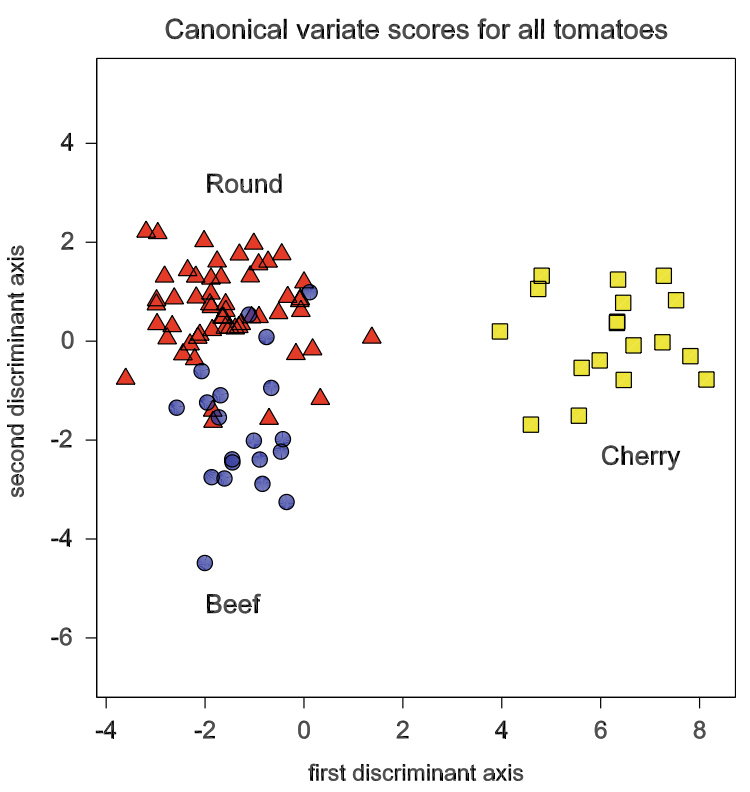

Fig. 10 Canonical variate scores for all tomatoes in twodimensions representing the two discriminant functions from the discriminant analysis of tomato type on all metabolites. Symbols represent tomato types (triangle $=$ round, circle $=$ beef and square = cherry) 
Table 1 Correlations between metabolite abundance and discriminant function scores (discriminant loadings) for all metabolites with the two discriminant functions (column a for function 1 , column $\mathrm{b}$ for function 2 )

Correlations between metabolites and discriminant functions

\begin{tabular}{ll}
\hline (a) First function & (b) Second function
\end{tabular}

\begin{tabular}{|c|c|c|c|}
\hline Phenylethanol & 0.39 & 2-Isobutylthiazol & 0.33 \\
\hline Glucose & 0.36 & $\begin{array}{l}\text { 6-Methyl-5hepten2- } \\
\text { one }\end{array}$ & 0.28 \\
\hline Sucrose & 0.35 & Methylsalicylate & -0.26 \\
\hline Fructose & 0.35 & Glucose & 0.25 \\
\hline 1-Penten-3-one & 0.31 & Fructose & 0.23 \\
\hline Citric-acid & 0.29 & Sucrose & 0.22 \\
\hline trans-2-Hexenal & 0.25 & cis-3-Hexenol & -0.19 \\
\hline Phenylacetaldehyde & 0.22 & 2-Methylbutanal & 0.18 \\
\hline Malic-acid & -0.21 & 2-Methoxyphenol & -0.16 \\
\hline cis-3-Hexenal & 0.20 & Myo-inositol & 0.14 \\
\hline trans-2-Heptenal & 0.19 & 1-Penten-3-one & 0.13 \\
\hline Myo-inositol & 0.16 & Phenylethanol & -0.13 \\
\hline cis-3-Hexenol & 0.15 & 3-Methylbutanol & 0.12 \\
\hline 2-Isobutylthiazol & -0.15 & Malic-acid & 0.09 \\
\hline $\begin{array}{l}\text { 6-Methyl-5hepten2- } \\
\text { one }\end{array}$ & -0.10 & $\beta$-Damascenone & -0.08 \\
\hline Hexanal & 0.07 & Phenylacetaldehyde & -0.07 \\
\hline Aspartic-acid & -0.06 & 2-Methylbutanol & -0.05 \\
\hline 2-Methylbutanal & -0.05 & trans-2-Hexenal & 0.05 \\
\hline$\beta$-Damascenone & 0.04 & $\beta$-Ionone & -0.04 \\
\hline 2-Methoxyphenol & -0.03 & Citric-acid & 0.04 \\
\hline$\beta$-Ionone & 0.03 & Glutamic-acid & -0.04 \\
\hline 2-Methylbutanol & -0.03 & cis-3-Hexenal & 0.03 \\
\hline 3-Methylbutanol & -0.02 & Aspartic-acid & 0.03 \\
\hline Methylsalicylate & -0.02 & trans-2-Heptenal & 0.01 \\
\hline Glutamic-acid & -0.01 & Hexanal & 0.01 \\
\hline
\end{tabular}

In both columns metabolites are sorted from top to bottom by absolute correlation with the discriminant function concerned

total plot variation, respectively. The "metabolic" distance, expressed as Mahalanobis distance, between beef and round tomatoes was an order of magnitude smaller (5.55) than the distances between cherry and round (60.62), and cherry and beef (58.87).

\section{Discussion}

In this study we investigated taste sensation related metabolites that display variation in greenhouse tomatoes. Methoxyphenol, methylsalicylate and 2-isobutylthiazol

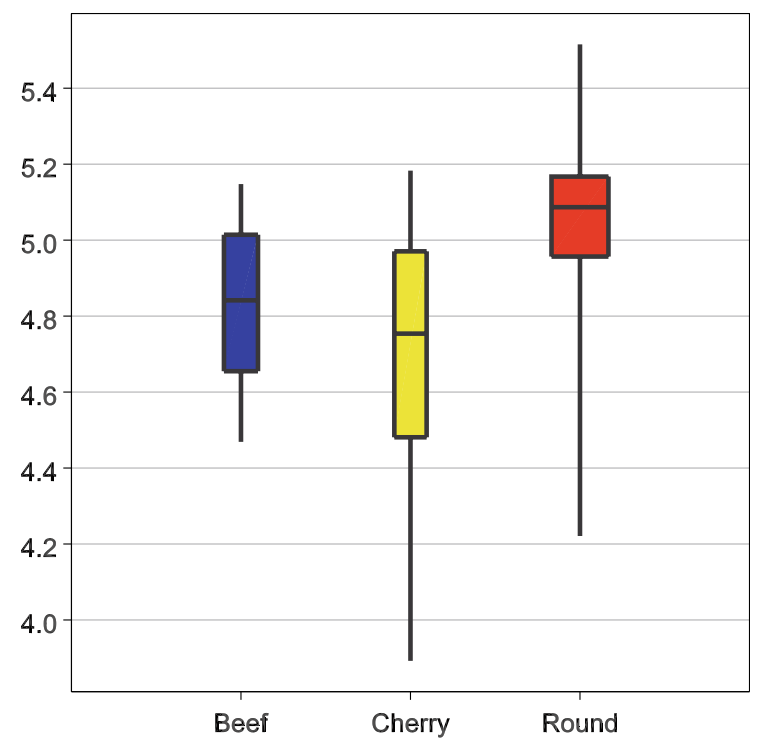

Fig. 11 Boxplot showing the variation in 2-isobutylthiazol within the three tomato type groups. Box width represents group size

phenylethanol had the largest variation, fructose, $\beta$-damascenone and glucose the smallest. The estimated heritability was highest for methylsalicylate and phenylethanol and lowest for the amino acids glutamic- and aspartic-acid. Some metabolites were always highly correlated, independent of the type of correlation considered. Examples of 'robust' clusters of correlated metabolites were methoxyphenol and methylsalicylate (phenylpropanoids), the phenolic metabolites phenylethanol and phenylacetaldehyde, the (iso)leucine derived metabolites 2- and 3-methylbutanol, methylbutanal, a cluster consisting of trans-2hexenal, cis-3-hexenal, 1-penten-3-one, cis-3-hexenol (lipid derived volatiles) and a glutamic- and asparticacid (amino-acids) group. All 'robust' relations seem to be direct relations, except for the relations that included cis-3-hexenol and the relation between trans2-hexenal and cis-3-hexenal. Many strong relations in the full phenotypic network were caused by tomato type related abundance and were therefore less clearly observable in the tomato-type corrected correlation network. An example is the relation between sugars and phenolic volatiles. Other metabolite relations were much more outspoken within a particular tomato type. In non-cherry tomatoes, for example, citric-acid and 2-isobutylthiazol were highly correlated to the methylbutanol/methylbutanal cluster, whereas in cherry 
tomatoes the methylbutanol/methylbutanal cluster was highly negatively correlated to the phenylpropanoid volatiles methylsalicylate and 2-methoxyphenol. The correlations within the sugar cluster were highly interdependent, only the glucose-fructose and a small fructose-myo-inositol relation were present in the partial correlation network.

In this paper, we used network plots to simultaneously visualize all significant correlations between metabolites. In contrast to PCA, network plots do not focus on representation of maximum variation in data matrices, which sometimes negatively affects representation of pairwise relations between variables. Especially, relations between metabolites whose variation is spread out over several principal axes can be easily overlooked in PCA biplots, even when metabolite abundance is normalized. We demonstrated that a fruitful way to explore patterns in metabolite variation is by a combination of PCA (without column standardization) and network plots. Correlation networks are very useful to study changes in metabolite relations when comparing different experimental conditions or different parts of the overall metabolic variation. By fixing the positions of the metabolite nodes, changes in correlation structure can be easily identified. Dissecting metabolite variation and correlations into identifiable genetic and environmental parts will help to understand relations of these metabolites to processes on other biological levels. Presently, we are investigating methods to link patterns in organoleptic variation to patterns in metabolic variation and molecular marker variation.

Acknowledgements This work was funded by the research program of the Center for BioSystems Genomics (project TQ3), which is a Centre of Excellence under the auspices of the Netherlands Genomics Initiative.

Open Access This article is distributed under the terms of the Creative Commons Attribution Noncommercial License which permits any noncommercial use, distribution, and reproduction in any medium, provided the original author(s) and source are credited.

\section{References}

Baldwin EA, Goodner K, Plotto A, Pritchett K, Einstein M (2004) Effect of volatiles and their concentration on perception of tomato descriptors. J Food Sci 69:S310-S318

Baldwin EA, Scott JW, Shewmaker CK, Schuch W (2000) Flavor trivia and tomato aroma: biochemistry and possible mechanisms for control of important aroma components. Hortscience 35:1013-1022

Batagelj V, Mrvar A (2003) Pajek-analysis and visualization of large networks. In: Jünger M, Mutzel P (eds) Graph drawing software. Springer, Berlin, p 77

Benjamini Y, Hochberg Y (1995) Controlling the false discovery rate-a practical and powerful approach to multiple testing. J Royal Stat Soc Ser B-Methodol 57:289-300

Burdon RD (1977) Genetic correlation as a concept for studying genotype-environment interaction in forest tree breeding. Silvae Genet 26:168-175

Buttery RG, Ling LC (1993) Volatile components of tomato fruit and plant-parts-relationship and biogenesis. Acs Symp Ser 525:23-34

Camacho DM (2005) The origin of correlations in metabolomics data. Metabolomics 1:53-63

Dillon WR, Goldstein M (1984) Multivariate analysis: methods and applications. Wiley, New York

Falconer DS, Mackay TFC (1996) Introduction to quantitative genetics. Longman, Harlow

Graffelman J, van Eeuwijk F (2005) Calibration of multivariate scatter plots for exploratory analysis of relations within and between sets of variables in genomic research. Biom J 47:863-879

Jolliffe IT (2002) Principal component analysis. SpringerVerlag, New York

Krumbein A, Peters P, Bruckner B (2004) Flavour compounds and a quantitative descriptive analysis of tomatoes ( $\mathrm{Lyc}$ opersicon esculentum Mill.) of different cultivars in shortterm storage. Postharvest Biol Technol 32:15-28

Malosetti M, Ribaut JM, Vargas M, Crossa J, van Eeuwijk FA (2008) A multi-trait multi-environment QTL mixed model with an application to drought and nitrogen stress trials in maize (Zea mays L.). Euphytica (this issue)

Morgenthal K, Weckwerth W, Steuer R (2006) Metabolomic networks in plants: transitions from pattern recognition to biological interpretation. Biosystems 83:108-117

Roessner-Tunali U, Hegemann B, Lytovchenko A, Carrari F, Bruedigam C, Granot D, Fernie AR (2003) Metabolic profiling of transgenic tomato plants overexpressing hexokinase reveals that the influence of hexose phosphorylation diminishes during fruit development. Plant Physiol 133:84-99

Ruiz JJ, Alonso A, Garcia-Martinez S, Valero M, Blasco P, Ruiz-Bevia F (2005) Quantitative analysis of flavour volatiles detects differences among closely related traditional cultivars of tomato. J Sci Food Agric 85:54-60

Steuer R (2006) On the analysis and interpretation of correlations in metabolomic data. Brief Bioinform 7:151-158

Steuer R, Kurths J, Fiehn O, Weckwerth W (2003a) Interpreting correlations in metabolomic networks. Biochem Soc Trans 31:1476-1478

Steuer R, Kurths J, Fiehn O, Weckwerth W (2003b) Observing and interpreting correlations in metabolomic networks. Bioinformatics 19:1019-1026

Storey JD, Tibshirani R (2003) Statistical significance for genomewide studies. Proc Natl Acad Sci USA 100:94409445

Tandon KS, Baldwin EA, Scott JW, Shewfelt RL (2003) Linking sensory descriptors to volatile and nonvolatile components of fresh tomato flavor. J Food Sci 68:2366-2371 
Tikunov Y, Lommen A, de Vos CHR, Verhoeven HA, Bino RJ, Hall RD, Bovy AG (2005) A novel approach for nontargeted data analysis for metabolomics. Large-scale profiling of tomato fruit volatiles. Plant Physiol 139:1125-1137

van Berloo R, Gort G, Zhu A, Ursem R, Bovy A, van Eeuwijk F (submitted) Association mapping for fruit quality traits in a set of cultivated tomato hybrids
Weckwerth W, Loureiro ME, Wenzel K, Fiehn O (2004) Differential metabolic networks unravel the effects of silent plant phenotypes. Proc Natl Acad Sci USA 101:7809-7814 